\title{
¿Son a priori los modelos explicativos de la selección
}

natural?*

Are Natural Selection Explanatory Models A Priori?

José Diez ${ }^{\dagger}$

Pablo Lorenzano ${ }^{\ddagger}$

\section{Resumen}

El estatus epistémico de la Selección Natural ha intrigado a biólogos y filósofos por igual, desde sus inicios hasta nuestros días. Un ejemplo contemporáneo prominente es Elliot Sober, quien afirma que la Selección Natural y otras teorías biológicas -y quizás económicas- son peculiares por incluir modelos explicativos/condicionales que son a priori en un sentido en el que los modelos explicativos/condicionales de la Mecánica Clásica y la mayoría de las demás teorías estándar no lo son.

En este artículo, analizando lo que creemos ser las cuatro posibles interpretaciones de la tesis de Sober, argumentamos que, dejando de lado las preferencias terminológicas, los posibles sentidos en los que los modelos explicativos de la Selección Natural pueden contar, o incluyen elementos que pueden contar, como a priori, también se aplican a la Mecánica Clásica y a otras teorías estándar, altamente unificadas.

Palabras clave: selección natural - Sober - modelos explicativos a priori - mecánica clásica

\begin{abstract}
The epistemic status of Natural Selection has intrigued to biologists and philosophers since the very beginning of the theory to our present times. One prominent contemporary example is Elliott Sober, who claims that Natural Selection, and some other theories in biology, and maybe in economics, are peculiar in including explanatory models/conditionals that are a priori in a sense in which explanatory models/conditionals in Classical Mechanics and most other standard theories are not.

In this paper, by analyzing what we take to be the four possible interpretations of Sober's claim, we argue that, terminological preferences aside, the possible senses in which explanatory models in Natural Selection can qualify, or include elements that can qualify, as a priori, also apply to Classical Mechanics and other standard, highly unified theories.
\end{abstract}

Keywords: natural selection - Sober - a priori explanatory models - classical mechanics

\footnotetext{
* Recibido: 25 de Julio de 2016. Aceptado con revisiones: 5 de Diciembre de 2016.

† Grupo de Investigación LOGOS, Universidad de Barcelona. Para contactar al autor, por favor, escribir a: diez.ja@gmail.com.

* Centro de Estudios de Filosofía e Historia de la Ciencia (CEFHIC), Universidad Nacional de Quilmes (UNQ)/CONICET. Para contactar al autor, por favor, escribir a: pablo.lorenzano@gmail.com.

$\$$ Este trabajo, que constituye una versión castellana abreviada de Díez \& Lorenzano (2015), desarrolla y elabora en detalle, introduciendo nuevos elementos esenciales, una objeción brevemente esbozada en Díez \& Lorenzano (2013). Quisiéramos agradecer a Daniel Blanco, Santiago Ginnobili, Carl Hoefer, Elliott Sober, Albert Solé y a dos referees anónimos por sus útiles comentarios a versiones previas a este artículo, que ha sido realizado con la ayuda de los proyectos de investigación FFI2012-37354 /CONSOLIDER INGENIO CSD20090056 (España), FFI2013-41415-P (España), PICT-2012 № 2662 (ANPCyT, Argentina) y PIP N 112-201101-01135 (CONICET, Argentina).

Metatheoria 8(1)(2017): 31-42. ISSN 1853-2322.

(c) Editorial de la Universidad Nacional de Tres de Febrero. Publicado en la República Argentina.
} 


\section{Introducción}

El estatus epistémico de la Selección Natural (SN, de aquí en adelante) ha intrigado a biólogos y filósofos por igual, tanto defensores como detractores de $\mathrm{SN}$, desde sus inicios hasta nuestros días. Un ejemplo contemporáneo prominente por parte de los defensores es Elliot Sober, quien afirma que enunciados causales a priori juegan un papel central en $\mathrm{SN}$ y otras teorías biológicas -y quizás económicas-. Además, Sober sostiene que modelos explicativos/condicionales específicos en SN son a priori en un sentido en el que modelos explicativos en Mecánica Clásica (MC, de aquí en adelante), y otras teorías estándar, no lo son (Sober 1993, 2008, 2011, Sober \& Fodor 2010).

En este trabajo, en primer lugar, formularemos la tesis de Sober y reconstruiremos algunos modelos explicativos particulares de SN, incluyendo sus ejemplos favoritos, tales como el modelo de proporción sexual de Fisher. Luego, discutiremos y evaluaremos la tesis de Sober de acuerdo con lo que consideramos son las posibles interpretaciones de 'modelo explicativo a priori'. Aquí discutimos tres interpretaciones centradas en modelos explicativos particulares, independientemente de su relación con un principio adaptativo general. En la interpretación final, discutimos la relación entre modelos explicativos particulares y un principio adaptativo general como un caso de relación de especialización dentro de redes teóricas basadas en principios-guía, mostrando que los aspectos epistémicamente difíciles son comunes a todas las teorías unificadas basadas en principios-guía. Concluimos que ninguna de estas interpretaciones hace sostenible la tesis de Sober: de acuerdo con algunas interpretaciones, $\mathrm{SN}$ contiene modelos explicativos a priori, pero también lo hace $\mathrm{MC}$; de acuerdo con otras, MC no contiene modelos explicativos a priori, pero tampoco lo hace SN. Así, en ausencia de una nueva interpretación, no hay un sentido en el cual modelos explicativos particulares son a priori en SN (y algunas otras teorías) pero no lo son en MC (y la mayoría de las teorías). Creemos que, aunque la posición de Sober sobre modelos a priori en SN no es dominante en el campo, el análisis que ofrecemos aquí no sólo completa de manera crucial algunas críticas que se le han hecho, sino que también ilumina aspectos de SN que han intrigado tanto a filósofos como biólogos desde sus comienzos hasta el presente.

\section{Lo que sostiene Sober y algunos ejemplos paradigmáticos}

Sober reconoce que hay un sentido en el cual todas las teorías empíricas hacen igual uso de modelos a priori, a saber: los modelos teóricos son definidos por medio de axiomas/leyes teóricos/as, de modo que tales modelos satisfacen a priori dichos axiomas/leyes. Estos modelos definidos a priori mediante axiomas teóricos son los usados por los enfoques semánticos para caracterizar las teorías empíricas como conjuntos de modelos (en todos estos enfoques, empero, las aserciones empíricas de las teorías, e.e. las afirmaciones de que cierta parte del mundo es subsumible en un modelo teórico, nunca son calificadas como a priori). Sin embargo, en diversos escritos Sober también afirma que hay otro sentido de acuerdo con el cual la Selección Natural (SN), y otras pocas teorías, hacen uso de modelos explicativos a priori, en tanto que la Mecánica Clásica (MC), y el resto de teorías empíricas estándar, no lo hacen (Sober 1993, 2008, 2011, Sober \& Fodor 2010; allí, Sober usa 'a priori' y 'no-empírico' como sinónimos). ${ }^{1}$ Esta segunda aserción es la que nos interesa aquí.

Sober (2011) comienza con un principio general de selección natural (PSN, así denominado en Lange \& Rosenberg 2011, p. 592) como caso de aserción adaptativa causal/explicativa que, según él, es a priori (Sober 2011, p. 575):

\footnotetext{
${ }^{1}$ Siguiendo a Sober, representamos a los modelos explicativos como abreviados por condicionales con el explanans como el antecedente y el explanandum como el consecuente. Dejamos de lado la rica literatura reciente y discusión sobre modelos, explicación y modelos explicativos en ciencia, ya que nada de lo que sigue depende de esta simplificación.
} 
PSN Si $A$ es más apto que $B$ en una población en la que no hay otras causas evolutivas en acción, y los rasgos son perfectamente heredables, entonces A incrementará su frecuencia.

Recordando la broma de Molière acerca de la virtus dormitiva, Sober defiende que tal principio es a priori, aunque, a diferencia de la virtus de Molière, científicamente relevante. Más aún, Sober sostiene que los modelos explicativos específicos en selección natural usan regularidades y aserciones causales que no son empíricos sino a priori: "La broma de Molière es una broma, pero los modelos causales a priori, que son parte de la teoría de la selección natural, no son asunto de risa” (Sober 2011, p. 573); "algunas afirmaciones causales son a priori. La broma de Molière provee un ejemplo trivial de una aserción causal a priori. Los modelos matemáticos de la selección natural proporcionan ejemplos no-triviales" (Sober 2011, p. 588).

Uno podría preguntarse si este componente a priori está involucrado en todas las teorías explicativas. Sober subraya que el uso de modelos explicativos a priori no es un rasgo general de todas las teorías explicativas (Sober 2011, pp. 581-582). Así, SN es peculiar en este aspecto, comparada con MC y otras teorías "comunes".

La tesis de Sober, entonces, es la siguiente:

(S) SN, y otras (¿pocas?) teorías contienen modelos explicativos (causales) a priori en un sentido en el que MC, y (muchas) otras teorías, no.

Como primer ejemplo específico simple Sober menciona el modelo cebra-león: "El modelo es a priori, de cualquier modo que sea representado" (Sober 2011, pp. 577-578). Y presenta el modelo de proporción de sexos como un caso representativo interesante, más complejo, de esta afirmación: "Pienso que la teoría de Fisher es una verdad matemática; el consecuente se sigue matemáticamente del antecedente una vez que todo se establece cuidadosamente" (Sober 2008, p. 45).

Nos referimos a estos dos casos en nuestra discusión de (S) como una tesis acerca de modelos explicativos específicos (regresaremos más adelante al PSN general), comenzando por el modelo de proporción de sexos que Sober mismo presenta como un paradigma suficientemente interesante de esta afirmación.

Proporción de Sexos: SI (1) en el contexto C, el cruzamiento es por azar

(2) en $\mathrm{C}$, costo por hijo $=$ costo por hija

(3) la reproducción de la proporción sexual es transmitida con variación al azar a lo largo de las generaciones

(4) el beneficio de la madre provisto por hijo/hija es la contribución reproductiva promedio del hijo/hija

(5) maximizar la energía reproductiva es beneficioso para la reproducción diferencial

ENTONCES (6) la proporción de sexos de nacimientos se aproxima a 50-50

(“ $x$ es beneficioso para la reproducción diferencial” significa que los individuos que realizan mejor la función $x$ tienden a incrementar su proporción en la población.) Éste es el modelo que explica la proporción sexual 50-50. Sustituciones diferentes de (1) o (2) implican explananda diferentes: si tenemos en su lugar $\left(1^{\prime}\right)$ cruzamiento hermano/hermana, entonces obtenemos ( $\left.6^{\prime}\right)$ la proporción sexual con desvío femenino. Cuando la segunda premisa es (2") que el costo es proporcional a la mortalidad, entonces obtenemos (6") la proporción sexual con desvío a la mortalidad. Otros cambios de (1) y (2) dan cuenta de otros explananda en diferentes modelos de proporción de sexos (véase Hamilton 1967). En los modelos SN de proporción sexual, (3)-(5) nunca cambian (más sobre esto, más adelante). 
Si reconstruimos el modelo cebra-león de un modo similar, obtenemos:

Cebra León: SI (1) en el contexto C las cebras son depredadas sólo por los leones

(2) la velocidad de las cebras se transmite con variación al azar a la siguiente generación

(3) las cebras escapan de los leones corriendo

(4) escapar de los predadores es beneficioso para la reproducción diferencial

ENTONCES (5) la proporción de cebras rápidas aumenta en $\mathrm{C}$ en relación con las cebras lentas

El modelo de proporción de sexos explica el rasgo relevante (proporción 50-50) como un caso de adaptación de fecundidad. El modelo cebra-león explica su rasgo relevante (velocidad) como un caso de adaptación de supervivencia, en este caso vía la mejora de la conducta beneficiosa consistente en escapar del predador incrementando la velocidad.

Sin embargo, no todas las adaptaciones por medio de la mejora de la conducta de escapar del predador involucran el incremento de la velocidad. Otra manera típica de mejorar la huida de los predadores es mediante camuflaje, como en el caso de las polillas negras. Pero no todas las adaptaciones de supervivencia son de tipo de escape de predadores; hay otros tipos, tales como el de comida/provisión de energía, como en el caso del cuello de la jirafa. Y por supuesto también hay adaptaciones que no se obtienen por medio de facilitar la supervivencia, sino p.e. de facilitar el cruzamiento, como en el caso de la cola del pavo real. Todos estos casos podrían reconstruirse de manera similar a como fueron reconstruidos los modelos de la proporción de sexos y del modelo cebraleón. Sin embargo, debido a razones de espacio, no lo haremos aquí. Lo que haremos a continuación será explicitar las diferentes posibles interpretaciones de la expresión "modelo explicativo a priori", que consideran a los condicionales explicativos de manera aislada, y analizar (S) de acuerdo con ellas, concluyendo con una cuarta interpretación que considera los condicionales explicativos en relación con principios-guía generales.

\section{Condicionales explicativos considerados de manera aislada}

La primera interpretación posible de (S) es sugerida por el pasaje citado antes: "[...]; el consecuente se sigue matemáticamente del antecedente una vez que todo se establece cuidadosamente" (Sober 2008, p. 45; nuestro énfasis; véase también Sober 1993). Según esta interpretación, (S) afirmaría que los modelos $\mathbf{S N}$ son condicionales en los que el antecedente implica lógicamente/matemáticamente al consecuente. Sin embargo, la reacción inmediata es sostener que esto no es específico de $\mathrm{SN}$ comparado con, digamos, MC: dadas ciertas condiciones iniciales, junto con (una aplicación específica de) la Segunda Ley y otros ciertos supuestos empíricos, inferimos a priori, p.e., las regularidades de la caída libre de Galileo, o el movimiento planetario de Kepler, o la trayectoria del péndulo, etc. Si reconstruimos el modelo explicativo MC para la ley de caída libre de Galileo, obtenemos el siguiente condicional:

Caída Libre Tierra: SI (1) no hay fricción

(2) velocidad inicial cero

(3) $m g=m d^{2} s / d t^{2}$

ENTONCES (4) $s=1 / 2 \mathrm{gt}^{2}$

en donde (4) se sigue lógicamente/matemáticamente de (1)-(3).

Sober (2011, pp. 581-582) reconoce esta objeción, pero sostiene que "la situación en la teoría evolutiva es diferente. Los modelos que he descrito son a priori; ellos tienen la forma 'Si IB, entonces P'; no hay ley empirica en el antecedente" (Sober 2011, p. 582; énfasis nuestro). 
Creemos que hay tres (o cuatro, si contamos la forma debilitada de la segunda) posibles interpretaciones del enunciado crucial "[los modelos explicativos en teoría evolutiva] tienen la forma 'Si IB, entonces P'; no hay ley empírica en el antecedente”. A continuación, analizaremos dos de ellas (la segunda también en su forma debilitada), que consideran a los modelos explicativos de manera aislada. Dejaremos para la próxima sección la última de las interpretaciones, que los vincula con un principio SN general.

Según la primera interpretación, habría que leer (S) como sigue:

(S1) SN, y (pocas) otras teorías, contienen modelos explicativos (causales) a priori en un sentido en el que MC, y (muchas) otras teorías no, a saber: los modelos $\boldsymbol{S N}$ son condicionales de la forma 'Si I $\mathscr{E} B$, entonces $P$ ', en los cuales el antecedente implica lógicamente/matemáticamente al consecuente y contiene sólo I y $B$, y nada más.

Pensamos que (S1) no puede ser verdadera. Más aún, entra en conflicto con lo que el propio Sober sostiene sobre cuestiones relacionadas. Si lo que Sober quiere decir con (S) fuera (S1), entonces Fodor (2008a, 2008b, Fodor \& Piattelli 2010a, 2010b) realmente habría establecido un punto fuerte: distintos modelos explicativos en SN no tendrían nada (adaptativo) en común, ya que I y B cambian de una explicación a otra. Sin embargo, en su discusión con Fodor, Sober (2008, Sober \& Fodor 2010) insiste correctamente en que los distintos modelos explicativos adaptativos comparten componentes adaptativos que son explicativamente esenciales.

Y Sober tiene razón, y Fodor está equivocado, precisamente porque (S1) no puede ser verdadero: hemos visto, p.e., que la explicación de Fisher hace uso esencial de Proporción de Sexos (4)-(5), compartido por todos los modelos de proporción de sexos, sin los cuales la derivación no tendría lugar, pero ni (4) ni (5) son condiciones iniciales o de contorno.

Proporción de Sexos (3) tampoco cambia en los modelos de proporción de sexos, pero podría contar como una condición general inicial común a todos los modelos de proporción de sexos (de hecho, una condición de transmisibilidad-con-variación del rasgo relevante está incluida en todos los modelos SN). Esto no es así en el caso de Proporción de Sexos (4) y (5). No son condiciones iniciales ni de contorno. Y lo mismo se aplica a las dos últimas premisas en Caida Libre Tierra.

Aunque tenemos una propuesta particular para el estatus de estas cláusulas que defenderemos más adelante, consideramos de momento incontrovertido que no son condiciones iniciales ni de contorno. Ya que son indispensables para la implicación lógica/matemática, (S1) no puede entonces ser verdadero. El antecedente del condicional que implica lógicamente/matemáticamente al explanandum, no puede contener únicamente condiciones iniciales o de contorno, ni en MC, ni en SN, ni en ninguna otra teoría.

La segunda interpretación surge de considerar el condicional al cual se refiere Sober como sólo conteniendo $I$ y $B$ en su antecedente, no como verdadero lógicamente/matemáticamente, sino sólo como verdadero a priori:

(S2) SN, y (pocas) otras teorías contienen modelos explicativos (causales) a priori en un sentido en el que $\mathbf{M C}$, y (muchas) otras teorías no, a saber: los modelos $\boldsymbol{S N}$ son condicionales de la forma 'Si I $\mathcal{E} \mathrm{B}$, entonces P', en los cuales el antecedente implica a priori (si bien no lógicamente/matemáticamente) al consecuente y contiene sólo I y $B$, y nada más.

¿Cómo evaluar (S2)? Tomemos un condicional verdadero a priori, aunque no lógicamente, tal como "SI Juan es soltero, ENTONCES Juan es no-casado". Existe algún contenido adicional, a saber: "los solteros son no-casados", el cual es a priori y tal que, añadido al antecedente, hace al condicional resultante "SI Juan es soltero \& los solteros son no-casados, ENTONCES Juan es no-casado" verdadero lógicamente/ matemáticamente. Generalizando: si SI X, ENTONCES Y (con X e Y no siendo a priori) 
es verdadero a priori pero no lógicamente/matemáticamente, entonces hay una $Z$ tal que SI $\chi \& \&$, ENTONCES $Y$ es verdadero a priori lógicamente/matemáticamente, y (si $Z$ sola no implica $Y$ ) $Z$ es a priori. En esta interpretación Sober se compromete a decir que, en modelos explicativos en SN, una vez el antecedente está completamente reconstruido para que implique lógicamente/matemáticamente al explanandum, las condiciones del antecedente que no son ni condiciones iniciales ni de contorno son ellas mismas a priori.

Según esta interpretación, Sober se comprometería a decir que, en los condicionales SN verdaderos lógicamente/matemáticamente anteriores, todos los componentes en los antecedentes que no son condiciones iniciales ni de contorno, son cognoscibles a priori -en un sentido en el cual los correspondientes componentes en los condicionales MC no lo son-. Encontramos difícil de defender esta afirmación. El hecho de que las cebras (y otros animales) escapen de los predadores corriendo, no parece ser cognoscible a priori en ningún sentido razonable del término. Y si uno quisiera sostener que tales hechos son a priori, entonces, sin argumento adicional, no habría razón para no aplicar la misma convención terminológica a los componentes que no están en I ni en B del antecedente de los modelos explicativos de MC. De modo que (S2) tampoco le sirve.

Una última opción es debilitar (S2) afirmando que, aunque no todos los componentes no-I\& $B$ sean a priori, algunos lo sean, en particular el último componente del antecedente en nuestras reconstrucciones, el que establece que alguna función es beneficiosa para la reproducción diferencial; y que ello no es el caso en MC y teorías similares. Pero, ¿son a priori las condiciones de la forma "...es beneficiosa para la reproducción diferencial”? No, no lo son. No son cognoscibles a priori en el sentido de que su justificación es independiente de la información empírica. Su justificación sí depende de información empírica. Que machos más fuertes se reproduzcan más, es algo que se aprende de la experiencia, y análogamente respecto de escapar de predadores o proveer comida o atraer a parejas sexuales. La conexión entre las funciones/conductas específicas y la reproducción diferencial a largo plazo no es a priori. Así, en los modelos explicativos SN particulares, las condiciones no-I\& $B$, todas las condiciones no-I\& $B$, son empíricas.

Esto agota las posibles interpretaciones de (S) que consideran a los modelos explicativos de manera aislada. Hay una posible interpretación más, relacionada con lo que creemos es la correcta lectura de las condiciones no-I\&B del antecedente, que vincula los modelos explicativos particulares con un principio SN general.

\section{Condicionales explicativos y principios-guía}

Como hemos visto, en los condicionales que resumen los modelos explicativos de $\mathrm{SN}$, el antecedente que implica lógicamente/matemáticamente el explanandum incluye esencialmente, además de $I$ \& $B$, algo más, $Z$, que no es a priori. El estatus empírico de este componente $Z$, aunque a posteriori, difiere en algunos aspectos relevantes de los de I y B. Sobre la base de esta diferencia, se podría calificar a estos modelos (con cierta heterodoxia terminológica) como a priori. Sin embargo, argumentaremos que en este respecto tampoco hay diferencia respecto de $\mathbf{M C}$.

¿Cuál es el estatus de este componente Z? Tómese p.e. Proporción de Sexos (4) y (5). Proporción de Sexos (5) es un hecho adaptativo general, común a todos los modelos de optimización, en este caso aplicado a la optimización de reproducción-energía. Proporción de Sexos (4) especifica, para este caso particular, en qué consiste el beneficio-energía. Como vimos, ambos son esenciales para la derivación y ninguno es una condición inicial o de contorno. Según nuestra propuesta, Proporción de Sexos (4) y (5) expresan conjuntamente la aplicación particular, al explanandum concreto 50-50 de proporción de sexos, de un principio SN adaptativo general, que llamamos el principio-guía de SN (PGSN, de aquí en adelante, muy similar al PSN mencionado más arriba): 
PGSN: Un rasgo transmisible (heredable) más apto t, e.e. que en un contexto $C$ facilita la realización de una función/conducta adaptativa, incrementa, ceteris paribus, su frecuencia en la población en $\mathrm{C}$.

Más brevemente: los rasgos transmisibles más aptos son beneficiosos para la reproducción diferencial (positiva).

Sostenemos que, sea cual sea la formulación del principio general SN, Proporción de Sexos (4) y (5) expresan la aplicación particular o especificación de ese principio al explanandum particular de proporción de sexos.

Cada modelo explicativo/condicional SN particular especifica, junto con las condiciones I \& B, el rasgo $t$ en cuestión y una función/conducta $f$ que (i) es beneficiosa para la reproducción diferencial y (ii) es realizada mejor con el rasgo que sin él (esta propuesta coincide, creemos, con la defendida por Brandon 1996, pp. 51-52).

En el caso de Proporción de Sexos: $t$ es la conducta reproductiva particular 50/50 de proporción de sexos y la función $f$ es la optimización de la energía reproductiva, especificada en (5), junto con lo que cuenta como el valor relevante a optimizar, e.e. el beneficio especificado en (4). Así, (4) y (5) especifican conjuntamente, para el caso particular del explanandum 50/50 de proporción de sexos, la función/conducta relevante que se deja abierta en PGSN y se afirma que es beneficiosa para la reproducción diferencial. Es en este sentido que PGSN "aparece implícitamente" en modelos SN particulares: los componentes no-I\&B introducen las condiciones esencialmente adaptativas que especifican el rasgo y la función adaptativa en cuestión.

Sostenemos, así, que modelos explicativos $\mathrm{SN}$ particulares son de la forma "Si $I \& B \&$ \& entonces $P$ ”, en donde $Z$ es una aplicación particular del PGSN general para el explanandum particular en cuestión. Esto provee un sentido en el cual $Z$ podría calificar derivativamente como a priori: si uno acepta (como lo hacemos) un sentido de 'a priori' de acuerdo con el cual el principio adaptativo general PGSN (o PSN, o su versión preferida del principio adaptativo general) es a priori, entonces uno podría decir derivativamente que los modelos explicativos particulares son a priori en el sentido que sus antecedentes incluyen un componente $Z$ que, aunque no a priori él mismo, es una aplicación particular-especificación del principio general a priori al explanandum en cuestión.

Esto proporciona una última interpretación de 'modelo a priori' y de (S):

(S3) SN, y (pocas) otras teorías contienen modelos explicativos (causales) a priori en un sentido en el que MC, y (muchas) otras teorías no, a saber: los modelos $\boldsymbol{S N}$ son condicionales de la forma 'Si I $\mathcal{G} B$ y $Z$, entonces $P$ ', en los cuales el antecedente implica lógicamente/matemáticamente al consecuente y $Z$ es una aplicación particular, al explanandum en cuestión, de un principio-guía a priori general

Sin embargo, si éste es el sentido en el que uno afirma que los modelos explicativos de selección SN son (derivativamente) a priori, tampoco se sostiene la tesis de Sober, ya que el estatus epistémico de PGSN, y de sus aplicaciones-especializaciones particulares en modelos explicativos particulares, no es exclusivo de SN respecto de MC y otras teorías, sino característico de toda teoría altamente unificada en cualquier ciencia.

El primero en llamar la atención sobre esta característica de principio-general-más-relación de especificación como esencial a todas las teorías altamente unificadas, fue Kuhn, tomando precisamente a MC como ejemplo paradigmático (Kuhn 1974, p. 465, 1970a, p. 155, 1970b, p. 272). Esta idea kuhniana ha sido elaborada en detalle por el estructuralismo metateórico con las nociones de especialización y de red teórica, y ha sido aplicada a muchas teorías unificadas. ${ }^{2}$

\footnotetext{
${ }^{2}$ Véase Balzer, Moulines y Sneed (1987) para una presentación completa, y Díez y Lorenzano (2002) o Moulines (2002) para una presentación sucinta de esta concepción metateórica.
} 
Por ejemplo, la red teórica de $\mathrm{MC}$ se ve (en determinado momento histórico) como sigue (aquí se muestran algunos nodos terminales que bastan para nuestra ejemplificación):

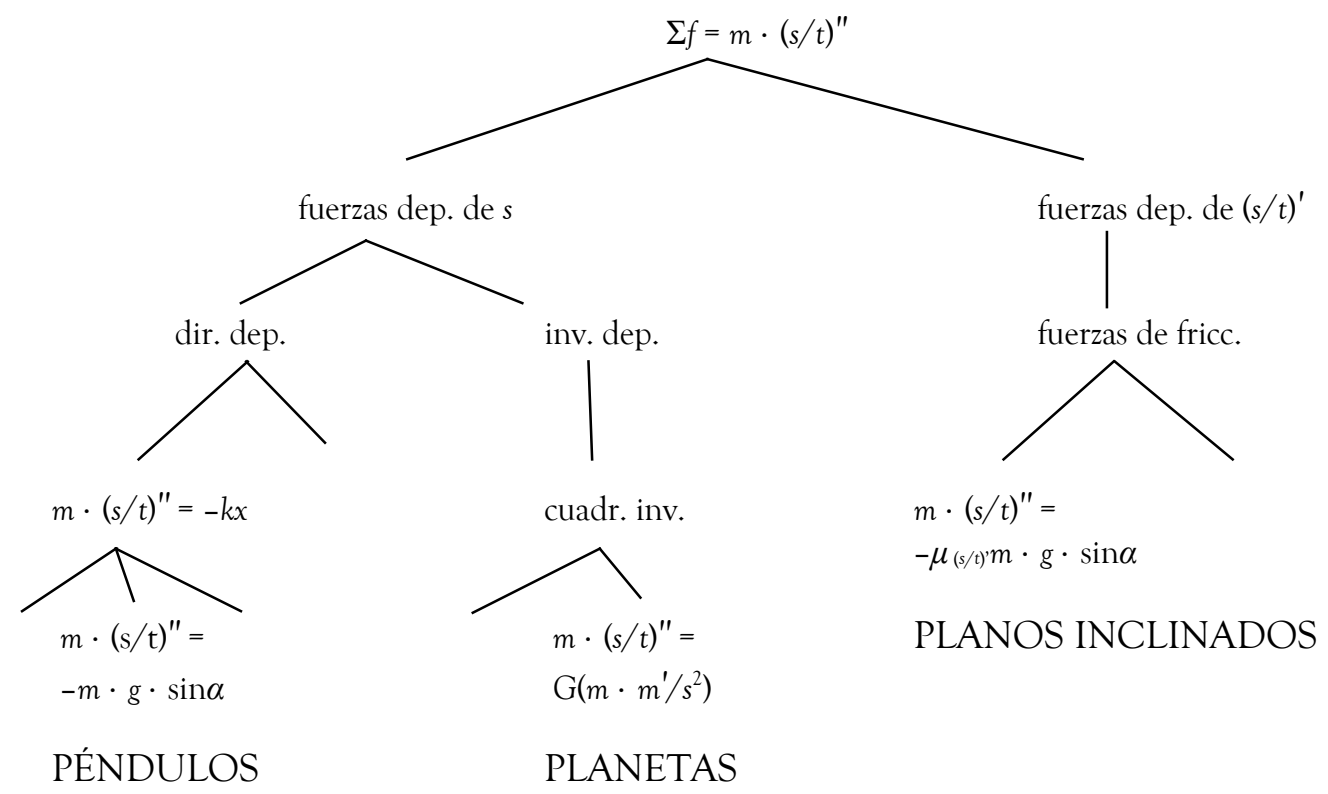

La red tiene a la Segunda Ley de Newton como el componente nomológico unificador en la cúspide, e.e. como el Principio-Guía de la Mecánica Clásica (o PGMC):

PGMC Para una trayectoria mecánica de una partícula con masa $m$, el cambio en la cantidad de movimiento, e.e. $m \cdot a$, se debe a la combinación de las fuerzas que actúan sobre la partícula.

El principio-guía de $\mathrm{MC}$ en la cúspide se especializa abriendo distintas ramas para distintos fenómenos/explananda. Esta especificación/ramificación se reconstruye en diferentes pasos: primero, fuerzas dependientes del espacio versus fuerzas dependientes de la velocidad; luego la rama dependiente del espacio se especializa en directa e inversamente dependiente del espacio; la rama directamente dependiente del espacio se especializa a su vez en lineal-negativamente dependiente del espacio y...; la rama inversamente dependiente del espacio se especializa cuadrático-inversamente y...; al final de cada rama tenemos una ley completamente especificada que es la versión del principio-guía para el fenómeno específico en cuestión: péndulos, planetas, planos inclinados, etc.

La relación arriba-abajo no es una relación de implicación o derivación, sino de especialización en el sentido estructuralista (Balzer, Moulines \& Sneed 1987, cap. IV): las leyes inferiores son versiones específicas de sus superiores, e.e. especifican algunos parámetros y dependencias funcionales que son dejadas parcialmente abiertas en las leyes anteriores en la rama.

Pues bien, sostenemos que SN tiene una estructura similar, con PGSN como su principio-guía unificador en la cúspide, especializándose hacia distintos tipos de generalizaciones adaptativas más específicas: para funciones de supervivencia, de cruzamiento, de fecundidad. Éstas, a su vez, se especializan en funciones más específicas: proveer comida, escapar de predadores,... como distintas funciones de supervivencia; atraer parejas sexuales, competir con rivales sexuales,... como distintas funciones de cruzamiento, etc. El nodo de escapar de predadores se especializa a su vez mediante distintas funciones, tales como incrementar la velocidad, mejorar el camuflaje, y así sucesivamente. La 
red teórica tiene aproximadamente la siguiente estructura (Díez \& Lorenzano, 2013; para una reconstrucción más completa, véase Ginnobili 2010): ${ }^{3}$

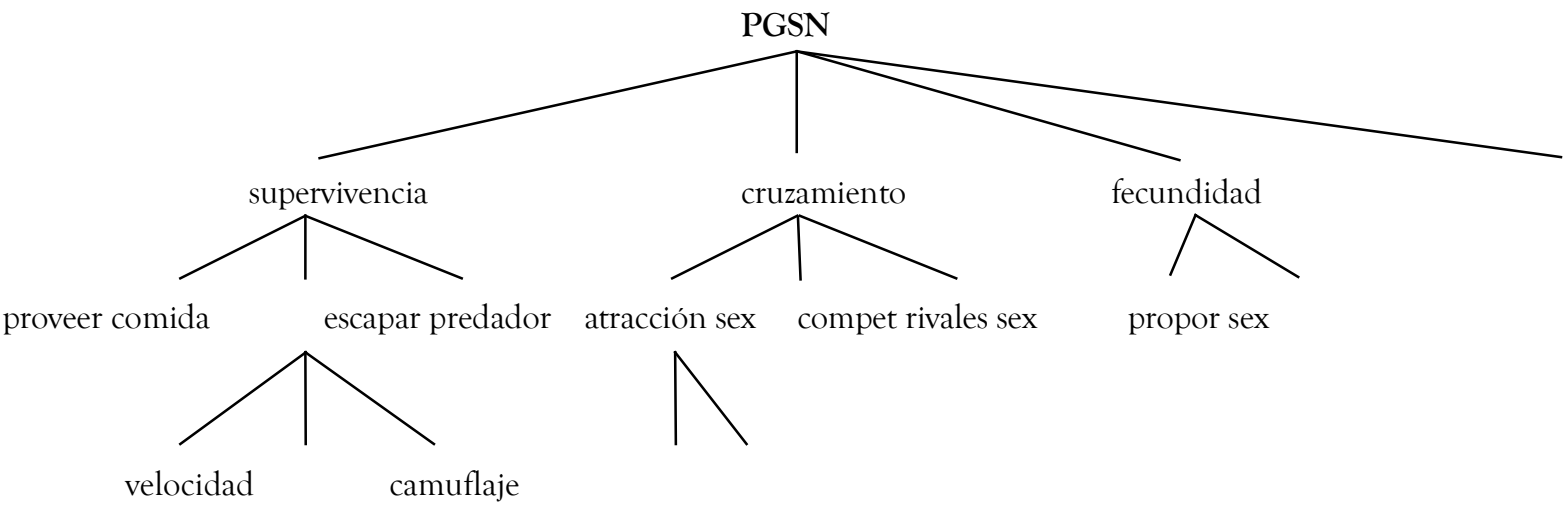

Al final de esta red encontramos modelos explicativos SN particulares, tales como Proporción de Sexos, Cebra León y los casos de las polillas negras, del cuello de la jirafa y de la cola del pavo real. En todos estos modelos encontramos la forma "Si $I \& B \& Z$, entonces $P$ ", con $Z$ especificando regularidades adaptativas particulares que especializan el PGSN general.

Este esbozo basta para subrayar las similitudes entre $\mathrm{SN}$ y MC, y otras teorías unificadas, relevantes para nuestro caso. Los modelos explicativos particulares en $\mathrm{MC}$ al final de la red teórica de la mecánica clásica, tales como Caída Libre Tierra, también muestran la misma forma "Si I \& B \& Z, entonces P", con el componente nomológico $Z$ que especializa el principio-guía general de MC (Caída Libre Tierra (3) es precisamente la forma que, de acuerdo con Kuhn, toma la Segunda Ley de Newton para la caída libre). Y encontramos una estructura similar en otras teorías unificadas, no sólo en física, sino también en biología, tal como la Genética Mendeliana (Lorenzano 2000).

(S3), por tanto, tampoco es correcta: el sentido en el cual los modelos SN pueden calificar derivativamente como a priori por incluir un componente $Z$ que (no siendo de $I$ ni de $B$ ) es una especialización del principio-guía a priori PGSN, también se aplica a los modelos explicativos de MC y de todas las teorías unificadas basadas en un principios-guía.

La única alternativa para defender (S3) es sostener que hay una diferencia con respecto a la aprioricidad entre los principios-guía mismos; esto es, que PGSN es a priori, pero PGMC no. En tal caso, los modelos SN podrían calificar derivativamente como a priori, mientras que los modelos $\mathrm{MC}$ no podrían. Esto tampoco es así. Por razones de espacio, sin embargo, no discutiremos esto aquí (véase Díez \& Lorenzano 2015).

\section{5. ¿Difieren PGSN y PGMC respecto de la aprioricidad?}

Si entendemos 'a priori' en un sentido que se aplica, además de a implicaciones lógicas, sólo a casos del tipo "los solteros son no-casados", entonces PGMC no es a priori, pero tampoco lo es PGSN. El asunto es si hay un sentido más amplio, pero todavía aceptable, de 'a priori' que se aplique a PGSN, pero no a PGMC, en cuyo caso podría aducirse tal sentido para justificar alguna diferencia respecto de la aprioricidad entre MC y SN, y con ello defender cierta justificación indirecta de la tesis de Sober. Veamos rápidamente los mejores candidatos.

Justificable independientemente de la experiencia. De acuerdo con este sentido, la Segunda Ley de Newton no sería a priori ya que fue establecida con la ayuda de información empírica. Sin embargo, si

\footnotetext{
${ }^{3}$ La que figura aquí podría no ser la única manera de reconstruir la red teórica de la selección natural. De hecho, podría haber distintas formas alternativas de hacerlo. Sin embargo, las posibles reconstrucciones alternativas, a pesar de sus diferencias, coincidirían todas ellas en el asunto que aquí se discute.
} 
lo que importa es cómo fue realmente establecido el principio, no hay diferencia con relación al principio adaptativo general; Darwin también usó una gran cantidad de información empírica, como lo atestigua su trabajo. Y si lo que importa es si el principio puede ser establecido sin la ayuda de evidencia empírica, ambos necesitan apoyo empírico.

Constitutivo del concepto. Se podría decir que PGSN es a priori en el sentido en que es "constitutivo del concepto", es decir, constituye (quizás junto con otras cosas) el significado/contenido de los términos/conceptos SN teóricos. Acordamos en que los principios-guía son constitutivos de los conceptos teóricos (Díez 2002, Lorenzano 2006), y también que esto los califica como a priori en un sentido relevante (el sentido a priori relativo). Hay de hecho un amplio acuerdo en que las leyes de T constituyen el significado/contenido de los términos/conceptos T-teóricos (el desacuerdo reside en qué leyes y cómo). Pero incluso si uno se aparta de este acuerdo, y niega que (algunas) leyes de T constituyen los conceptos de T, lo que importa para nuestro caso es que se debe prima facie adoptar la misma posición general. Si uno está dispuesto a defender que las leyes de SN, y al menos PGSN, constituyen el concepto SN-teórico de aptitud, pero que ninguna ley de $\mathrm{MC}$, ni siquiera PGMC, constituye el concepto MC-teórico de fuerza, entonces la carga de la prueba está de su lado.

Revisabilidad. Otra opción sería sostener que se puede decir que PGMC no es a priori ya que ha sido revisado, mientras que PGSN lo es ya que no es revisable. Sin embargo, todo lo que sabemos es que PGSN no ha sido revisado todavía, lo cual no significa que no sea revisable. Y nuestros sentimientos actuales no importan en lo absoluto: durante casi dos siglos los científicos (y los filósofos) sintieron que los principios nucleares de Newton no eran revisables. Por otro lado, ya sabemos que los principios constitutivos de conceptos son revisables: que la combustión consiste en liberar una sustancia es constitutivo del concepto "flogisto" aunque fue por supuesto revisado y abandonado más de dos siglos atrás.

Irrestricción empírica y carácter heurístico. Otra opción es defender que PGSN es a priori en el sentido que es meramente programático; no dice "casi nada" empíricamente. Acordamos con ello, pero sostenemos que esto también se aplica a PGMC. Como sugiere Kuhn, y ha enfatizado el estructuralismo metateórico, los principios-guía, todos ellos, son epistémicamente peculiares en que no pueden ser contrastados empíricamente "de manera aislada", sino que pueden ser contrastados, y eventualmente falsados, sólo a través de una de sus versiones específicas para un fenómeno específico. En este sentido, los principios-guía son heurístico-programáticos: nos dicen el tipo de cosas (una fuerza dinámica específica, una función adaptativa específica) que debiéramos buscar cuando queremos explicar un fenómeno específico. Como promesa heurística, PGSN tiene una clara lectura programática: "Cuando un rasgo cambia en una población, busque su fuerza adaptativa: una función o conducta que, en el ambiente dado, mejora el éxito reproductivo y es mejor realizada con el rasgo que sin él”. Pero lo mismo hace PGMC: "Cuando una partícula con masa cambia su movimiento, busca fuerzas que, combinadas, den cuenta de su cambio en el movimiento". Tomados de manera aislada, sin sus especializaciones, los principios-guía dicen muy poco empíricamente. Pueden ser considerados, si se los toma de manera aislada, como "empíricamente irrestrictos" (Moulines 1984). Dado su carácter esquemático, con parámetros que se dejan abiertos, no pueden ser contrastados incluso con supuestos auxiliares, necesitan una especificación concreta de los parámetros, i.e. adoptar la forma de una ley especializada, a fin de ser contrastados (con los correspondientes supuestos auxiliares). Su cognoscibilidad es difícil, ya que necesitan investigación empírica para ser conocidos (no son definiciones explícitas, eliminables tales como "los solteros son hombres adultos no-casados" o "la aceleración es la segunda derivada del espacio sobre el tiempo"), pero son empíricamente irrestrictos al mismo tiempo. Uno puede calificar su peculiar estatus epistémico con el término que más le plazca, incluyendo 'a priori', pero lo importante es que, sea cual sea la terminología que uno prefiera, uno debe calificar a todos ellos, a PGSN, pero también a PGMC y a otros, del mismo modo.

Vacuidad. Se puede finalmente insistir en que "aún, PGSN parece ser más vacío, trivial” que PGMC. No creemos que sea así. PGMC nos dice que los cambios en cantidad de movimiento que $\mathbf{M C}$ 
acepta como explananda, deben ser explicados en términos de fuerzas específicas que tenemos que postular/descubrir. No creemos que esto sea menos vacío que decir que los cambios en rasgos en una población que cuenten como explananda de $\boldsymbol{S N}$, deben ser explicados en términos de conductas adaptativas y de rasgos más aptos. Es verdad que si concebimos las funciones/conductas como aquellas relevantes para la reproducción, lo que afirma PGSN considerado de manera aislada es muy poco, pero también es verdad que si concebimos las fuerzas mecánicas como aquellas relevantes para el cambio de trayectoria, lo que afirmamos con PGMC considerado de manera aislada es muy poco también. Sin sus especializaciones ambos principios parecen ser igualmente "vacíos", sólo nos dicen el tipo de cosas que debemos buscar; esto es muy poco, pero (contra Fodor) lo suficiente para hacerlos algo más, y más interesantes, que meros truismos del tipo "si algo sucede es a causa de algo". Y, nuevamente, el diagnóstico es el mismo para PGSN y para PGMC.

Así, en ninguno de estos sentidos PGSN califica como a priori y PGMC no. Con eso (y mientras el defensor de Sober no ofrezca otro sentido que haga al primero a priori y al segundo no) concluye nuestro análisis de (S3): (S) leído como (S3) es igualmente insostenible. Así, (S1), (S2) y (S3) son insostenibles. A falta de una interpretación alternativa, (S) es entonces insostenible.

\section{Conclusión}

Hemos mostrado que las distintas interpretaciones posibles de (S) analizadas hacen a (S) insostenible: en los sentidos de 'modelo a priori' en los que uno puede decir razonablemente que los modelos explicativos de SN son a priori, también lo son los modelos explicativos de $\mathbf{M C}$; en los sentidos en los que los modelos explicativos de $\mathrm{MC}$ no son a priori, tampoco lo son los modelos de SN.

Por lo tanto, en ausencia de una nueva interpretación distinta de "modelo explicativo a priori" que se aplica a modelos explicativos de SN, pero no de MC, (S) es insostenible.

Bibliografía

Balzer, W., Moulines, C.U. y J.D. Sneed (1987), An Architectonic for Science. The Structuralist Program, Dordrecht: Reidel. (Traducción al castellano de P. Lorenzano: Una arquitectónica para la ciencia. El programa estructuralista, Bernal: Universidad Nacional de Quilmes, 2012.)

Brandon, R.N. (1996), Concepts and Methods in Evolutionary Biology, Cambridge: Cambridge University Press.

Díez, J. (2002), “A Program for the Individuation of Scientific Concepts”, Synthese 130(1): 13-48.

Díez, J. y P. Lorenzano (2002), "La concepción estructuralista en el contexto de la filosofía de la ciencia del siglo XX", en Díez, J. y P. Lorenzano (eds.), Desarrollos actuales de la metateoría estructuralista: problemas y discusiones, Bernal: Universidad Nacional de Quilmes/Universidad Autónoma de Zacatecas/Universidad Rovira i Virgili, pp. 13-78.

Díez, J. y P. Lorenzano (2013), "Who Got What Wrong? Fodor and Piattelli on Darwin: Guiding Principles and Explanatory Models in Natural Selection", Erkenntnis 78(5): 1143-1175.

Díez, J. y P. Lorenzano (2015), “Are Natural Selection Explanatory Models A Priori?”, Biology E Philosophy 30(6): 787 809.

Fodor, J.A. (2008a), "Against Darwinism”, Mind and Language 23(1): 1-24.

Fodor, J.A. (2008b), "Replies", Mind and Language 23(1): 50-57.

Fodor, J.A. y M. Piattelli-Palmarini (2010a), What Darwin Got Wrong, London: Profile Books, $2^{\text {nd }}$ edition with an Afterword, New York: Picador.

Fodor, J.A. (2010b), "Misunderstanding Darwin, and Exchange", Boston Review, 17 de marzo. Accesible en: http://bostonreview.net/BR35.2/darwin_exchange.php\#reply. 
Ginnobili, S. (2010), "La teoría de la selección natural darwiniana”, Theoria 25(1): 37-58.

Hamilton, W.D. (1967), “Extraordinary Sex Ratios”, Science 156(28 April 1967): 477-488.

Kuhn, T.S. (1970a), The Structure of Scientific Revolutions, $2^{\text {nd }}$ edition, Chicago: University of Chicago Press.

Kuhn, T.S. (1970b), "Reflections on my Critics", en Lakatos, I. y A. Musgrave (eds.), Criticism and the Growth of Knowledge, Cambridge: Cambridge University Press, pp. 231-278.

Kuhn, T.S. (1974), "Second Thoughts on Paradigms", en Suppe, F. (ed.), The Structure of Scientific Theories, Urbana, Ill.: University of Illinois Press, pp. 459-482.

Lange, M. y A. Rosenberg (2011), “Can There Be A Priori Causal Models of Natural Selection?”, Australasian Journal of Philosophy 89(4): 591-599.

Lorenzano, P. (2000), "Classical Genetics and the Theory-Net of Genetics”, en Balzer, W., Moulines, C.U. y J.D. Sneed (eds.), Structuralist Knowledge Representation: Paradigmatic Examples, Amsterdam: Rodopi, pp. 251-284.

Lorenzano, P. (2006), "Fundamental Laws and Laws of Biology”, en Ernst, G. y K.-G. Niebergall (eds.), Philosophie der Wissenschaft, Wissenschaft der Philosophie, Paderborn: Mentis-Verlag, pp. 129-155.

Moulines, C.U. (1984), “Existential Quantifiers and Guiding Principles in Physical Theories”, en Gracia, J.J.E., Rabossi, E., Villanueva, E. y M. Dascal (eds.), Philosophical Analysis in Latin America, Dordrecht: Reidel, pp. 173. 198.

Moulines, C.U. (2002), “Structuralism as a Program for Modeling Theoretical Science”, Synthese 130(1): 1-12.

Sober, E. (1993), Philosophy of Biology, London: Oxford University Press.

Sober, E. (2008), “Fodor's Bubbe Meise Against Darwinism”, Mind and Language 23(1): 42-49.

Sober, E. (2011), “A Priori Causal Models of Natural Selection”, Australasian Journal of Philosophy 89(4): 571-589.

Sober, E. y J.A. Fodor (2010), “Discussion: Who Got What Wrong?”, bloggingheads.tv, 20 de marzo. Accesible en: http://bloggingheads.tv/videos/2492. 